\title{
From face images and attributes to attributes
}

\section{Conference Paper}

Author(s):

Torfason, Robert; Agustsson, Eirikur; Rothe, Rasmus; Timofte, Radu

Publication date:

2017

Permanent link:

https://doi.org/10.3929/ethz-a-010811115

Rights / license:

In Copyright - Non-Commercial Use Permitted

Originally published in:

Lecture Notes in Computer Science 10113, https://doi.org/10.1007/978-3-319-54187-7_21 


\title{
From face images and attributes to attributes
}

\author{
Robert Torfason, Eirikur Agustsson, Rasmus Rothe, Radu Timofte \\ Computer Vision Laboratory, ETH Zurich, Switzerland
}

\begin{abstract}
The face is an important part of the identity of a person. Numerous applications benefit from the recent advances in prediction of face attributes, including biometrics (like age, gender, ethnicity) and accessories (eyeglasses, hat). We study the attributes' relations to other attributes and to face images and propose prediction models for them. We show that handcrafted features can be as good as deep features, that the attributes themselves are powerful enough to predict other attributes and that clustering the samples according to their attributes can mitigate the training complexity for deep learning. We set new state-of-the-art results on two of the largest datasets to date, CelebA and Facebook BIG5, by predicting attributes either from face images, from other attributes, or from both face and other attributes. Particularly, on Facebook dataset, we show that we can accurately predict personality traits (BIG5) from tens of 'likes' or from only a profile picture and a couple of 'likes' comparing positively to human reference.
\end{abstract}

\section{Introduction}

Attributes are semantic features allowing for mid-level representations, between the low-level features and the high-level labels, with application to recognition of people, objects, and activities [1,2]. Face recognition and verification $[3,4]$ are two domains where attributes have been applied successfully. The facial at-

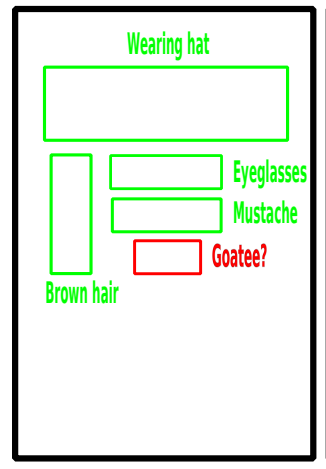

a)

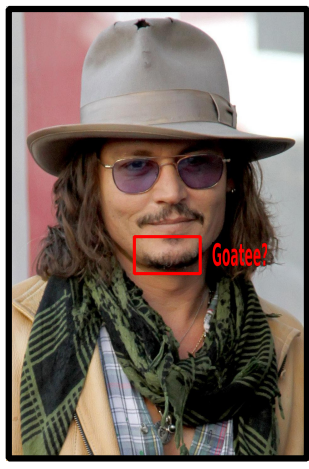

b)

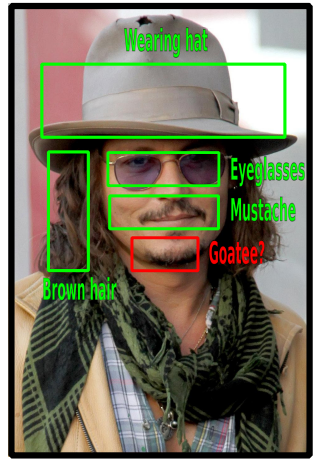

c)

Fig. 1. Attribute completion task: 'Goatee' attribute prediction from a) other attributes, b) face image, and c) combined face image and other attributes. 
tributes (see Figure 1) can include biometrics such as age, ethnicity, gender, but also particular categories distinguishing among, for example, hair colors, types of beards, or hair styles. Other attributes can capture the presence of accessories (such as earrings, eyeglasses, hat), makeup changes (lipstick), and mood/facial expressions. Behind each face is a distinct individual with attributes derived from preferences ('likes' for certain items from Facebook), CV (education, experience), habits, geo-locations, and even personality traits. The accurate prediction of such attributes is the key for the success of HCI applications where the computer application needs to 'read' the human by estimation of age, gender, and facial expression to interact accordingly. Visual data can be stored and searched using attributes [5], moreover, in cases when the visual data is insufficient the attributes might bring the extra information for more accurate recognition.

Attribute prediction is a challenging problem and while for biometrics the research spans over decades, the other attributes have been investigated less and, moreover, most of the recent research treats each attribute individually as a prediction from face image domain to the attribute label. The recent release of large-scale attribute datasets such as CelebA [6] (with face attributes) and Facebook BIG5 (with personality traits and 'likes') [7] fueled the recent advances and the interest resurgence for attributes as research topic. Convolutional Neural Networks (CNNs) proved their effectiveness on many computer vision tasks such as classification and end-to-end mappings [8-10]. The same is valid for attribute prediction where the recent top solutions rely on deep learning for representations and/or for direct attribute prediction $[1,6,11-14]$. We observe that generally the methods treat the prediction of each attribute individually starting from the face image even if there are clear dependencies between some attributes ('males' are unlikely to wear 'earrings' or 'lipsticks', 'females' to be 'bold' and have a 'goatee'). This leads to expensive solutions as the number of models and the training time are linear in the number of attributes and underachievement because of discarding important information - the strong dependencies.

We propose to make use of the strong dependencies among attributes. By doing so, we can robustly train deep features meaningful to all attributes. Furthermore, we can use attributes to predict other attributes and thus to solve the attribute completion task (see Figure 1). Note that with most of our settings we are capable to achieve state-of-the-art performance for most attributes of two recent large-scale datasets: CelebA [6] and Facebook BIG5 [7].

Our main contributions are as follows:

1. we propose clustering of attributes for efficient $\mathrm{CNN}$ representation learning;

2. we show that classic hand-crafted features can be as good as deep features and complementary;

3. we study the correlations between attributes for attribute completion with and without visual features;

4. we achieve state-of-the-art performance for most attributes on CelebA;

5. we achieve better than human references performance for personality traits prediction on Facebook BIG5 dataset by using a history of at least 20 'likes' and surprisingly good accuracy when using solely the profile picture; 
The paper is structured as follows. In Section 2 we review related works. In Section 3 we describe the datasets and experimental setup. In Section 4 we study the prediction of attributes when other attributes are known. In Section 5 we study the direct prediction of (facial) attributes from face images and propose clustering of attributes for representation learning. In Section 6 we analyze the performance when both visual and attributes are used for predicting other attributes. We discuss the results and conclude the paper in Section 7.

\section{Related work}

Visual attributes in their simplest and most common form are binary properties describing the image contents (see Figure 1). In general, any (image) property that can be quantified can be an attribute. For example, 'age' as biometric and 'personality trait score' of an user with an image.

Very recently, Liu et al. [6] show remarkable attribute prediction results using deep learning on large datasets (CelebA and LFWA) with thousands of face images annotated with 40 binary attributes. Youyou et al. [7] show that Facebook 'likes' can be used to predict the scores of BIG5 personality traits. Note that in this case the number of items/attributes that can be 'liked' is very large, while the 'likes' are very sparse and binary. One item is informative for a Facebook user only when is liked, otherwise is difficult to interpret as it needs additional information (did the user not like it on purpose? or was the user not aware of it?). The personality traits scores are real values. Both likes and personality scores are attributes for the Facebook user represented by a profile picture. We denote Youyou et al. 's dataset as Facebook BIG5 dataset. Prior Youyou et al. , Kosinski et al. [15] successfully predicted different user attributes (biometrics and traits) using Facebook data.

Visual attribute prediction is a multi-label classification (MLC) problem, where each image has multiple class labels / attributes. Most of the literature treats binary attributes individually (e.g. biometrics such as gender and ethnic group) in both modeling and prediction phases. This is known as Binary Relevance (BR) [16] which does not model correlations between binary labels and uses a number of models/classifiers in the number $L$ of labels/attributes. Pairwise classification (PW) [17] with $\frac{L(L-1)}{2}$ models/classifiers and label combination (LC) [18] with $2^{L}-1$ labels are other typical approaches which account for correlations but suffer from time complexity for large $L$ values. Recently, classifier chains (CC) [19] were shown to improve over PW while keeping in check the complexity.

Fine-Grained Image Classification (FGIC) is another direction closely related to our work. FGIC deals with class labels that are visually very similar, equivalent to, in attributes terms, images which differs in as few as 1 attribute when a (very) large number of attributes are available [20].

Multi-task learning (MTL) aims at solving problems with shared and/or related information [21] and is a very active direction in attribute prediction. Wang and Forsyth [22] learn jointly visual attributes, object classes and visual 
saliency in images with an iterative procedure and multiple instance learning. Wang and Mori [23] learn discriminative latent models for objects and attributes. Parikh and Grauman [24] propose to model relative attributes instead of binary attributes and to relate unseen object categories to seen objects through attributes, which is a form of zero-shot learning.

The visual attributes once predicted are generally used as medium-level semantic features with applications such as indexing and retrieval $[3,5]$, zero-shot classification of unseen objects based on attribute description $[25,26]$, and recognition [4].

\section{Experimental setup}

In our work we employ two complementary datasets and corresponding benchmarks as described below.

\subsection{CelebA face images and attributes dataset}

CelebA dataset was recently introduced by Liu et al. [6] for studying facial attributes in the wild. CelebA contains images of ten thousand celebrities, each with $\sim 20$ different images for a total of 202,599 images. Each image in CelebA is annotated with 40 binary facial attributes (such as 'Attractive', 'Male', 'Gray hair', 'bald', 'has beard', 'wearing lipstick', see Figure 2). Additionally, each image has 5 facial landmarks annotated, 2 for eyes, 1 for the nose and 2 for the corners of the person's mouth. In all our experiments we adhere to the benchmark setup from [6] such that we can directly compare our results on the test partition of the CelebA dataset with other found in the literature.

\subsection{Facebook profile images, likes and personality traits dataset}

Facebook BIG5 personality traits dataset was recently introduced by Youyou et al. [7] for studying the prediction of personality traits from the 'likes' history of the users of the Facebook social network. A 'like' shows a positive association of a user with online and offline items/subjects such as sport, musicians, products, celebrities, etc. However, the absence of a 'like' of a user for an item is not informative because the user equally could have a negative position, neutral position, positive but not expressed, or was not aware of that item within Facebook framework. The dataset has been collected by the myPersonality project ${ }^{1}$ and targets Facebook users and their attributes. Thousands of Facebook users willingly agreed to provide their 'likes' history and profile pictures, and to fill in a standard 100-item International Personality Item Pool (IPIP) Five-Factor Model of personality [27] questionnaire [28]. The personality traits (aka BIG5) measured by the 100 IPIP are: openness, conscientiousness, extraversion, agreeableness and neuroticism. Each trait gets a score on a fixed scale. The number

\footnotetext{
${ }^{1}$ http://mypersonality.org
} 


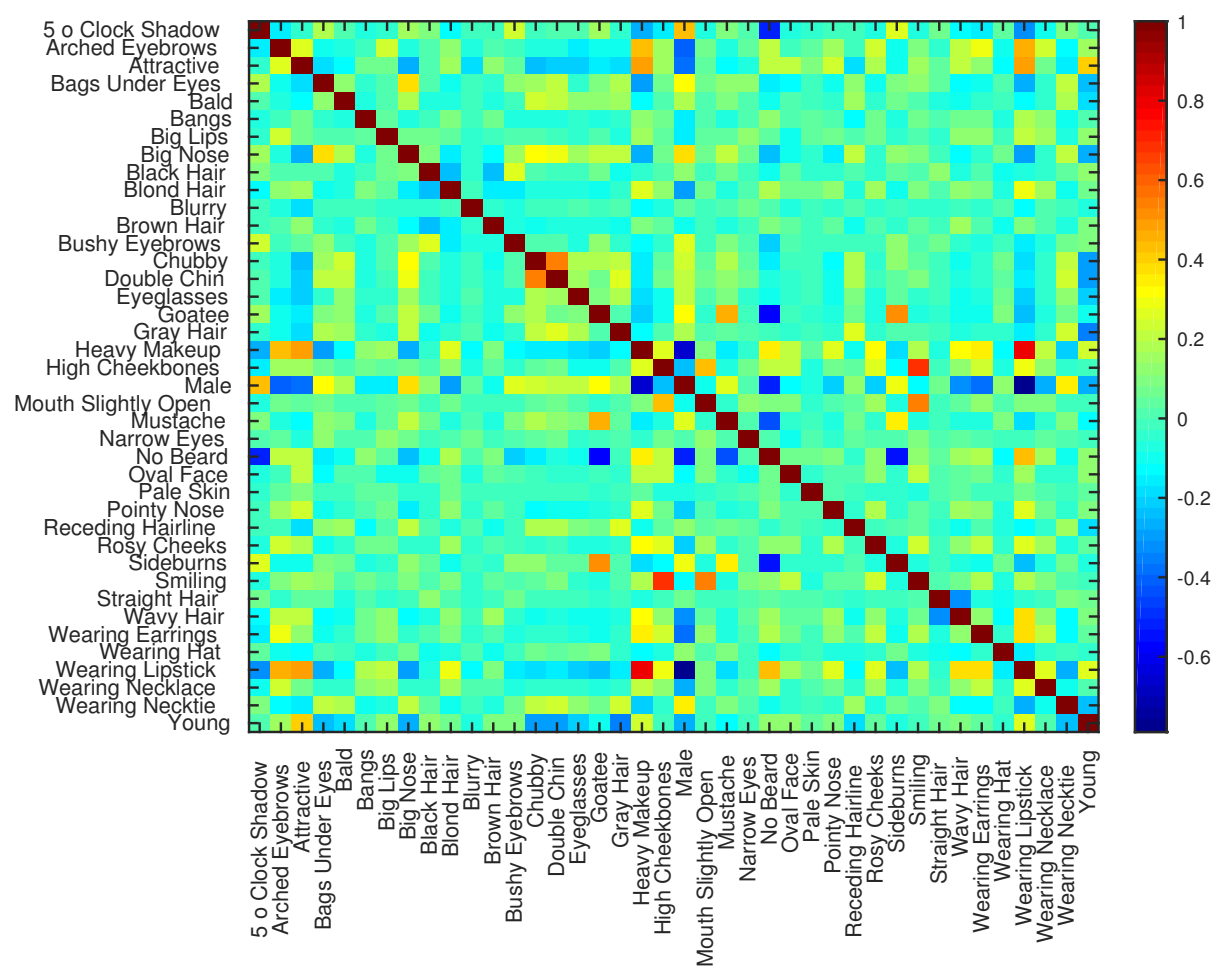

Fig. 2. Correlation between individual attributes in the CelebA dataset.

of users/samples that have results from the personality test, a history of likes and have profile images available make up the dataset 51,617 samples as used in our experiments. Note that in the original work of Youyou et al. [7] were used 70,520 samples (many without profile images) for experimental evaluation. For our experiments we keep the reporting setup from Youyou et al. [7] scaled to our lower number of samples. That is, we report Pearson correlation results computed with 10 fold cross-validation. The Pearson correlation is computed between the predicted personality trait score and the ground truth score as obtained based on the filled in 100 IPIP questionnaire by the Facebook user. The self-other agreement, human judgment references for work colleague, friend, family, spouse, humans' average are all reported as in [7].

\section{From attributes to attribute prediction}

When analyzing a face image, we may already know some of the attributes, e.g. based on the identity of the subject, descriptive text or other metadata. In such cases, we could leverage this information to predict the other attributes, i.e. solve the attribute completion task. This is one of the many cases and potential applications when it is desirable to be able to predict attributes or a set of attributes by using a limited set of other attributes. 


\begin{tabular}{l|ccccc||c} 
\# train attr. & 39 & 30 & 20 & 10 & 1 & flat prediction \\
\hline SVM & $\mathbf{8 7 . 4 4} \pm \mathbf{7 . 7 7}$ & $86.76 \pm 8.03$ & $85.98 \pm 8.65$ & $84.23 \pm 10.50$ & $80.76 \pm 14.27$ & $80.04 \pm 15.35$ \\
LASSO & $87.18 \pm 7.72$ & $87.11 \pm 8.18$ & $85.92 \pm 8.95$ & $84.20 \pm 10.32$ & $80.76 \pm 14.27$ & $80.04 \pm 15.35$
\end{tabular}

Table 1. CelebA prediction results (average accuracy [\%] and standard deviation) from subsets of attributes.

\subsection{CelebA face attributes}

Each CelebA image is labeled with 40 facial attributes. These attributes have predictive quality for each other due to their dependencies, e.g. 'wearing lipstick' correlates with 'heavy makeup' and 'young' with 'attractive'. The amount of annotated attributes and the total number of samples makes CelebA an ideal dataset to analyze the attribute completion task when starting from other attributes. In Figure 2 we show the matrix of correlations for the facial attributes of CelebA as found in the training data. We represent the samples by binary vectors corresponding to their known attributes at test. We use LASSO regression [29] followed by thresholding, or a linear SVM [30,31] for binary attribute prediction. We consider 5 cases/setups corresponding to the number of attributes that are known and used to build prediction models for the remaining attributes. We train models using $1,10,20,30$, and 39 attributes, resp., for predicting the remaining attributes, individually. For the case with 39 known attributes we train 40 SVM models and achieve a $87.44 \%$ average accuracy and 7.77 standard deviation at test. Table 4 summarizes how the predictive quality diminishes when smaller subsets of attributes are used to predict other attributes. The reported results were generated by taking 1500 random subsets of 10 attributes, 1000 random subsets of 20 attributes, and 500 random subsets of 30 attributes and the predictive results for the remaining 30,20 , and 10 corresponding attributes were averaged. When predicting 1 attribute from 39 and 39 attributes from 1, the number of permutations is computationally manageable and all different permutations are averaged.

In the setting where 1 attribute is predicted from 1 other attribute, the classifier in general learns the flat prediction. This is the case except for highly correlated features where the prediction improves slightly from the flat baseline prediction.

The average prediction accuracy of $87.44 \%$ achieved using solely attributes (39) to predict the missing attributes is remarkable as it compares favorably with many results from Table 2 .

\subsection{Facebook BIG5 personality traits}

Each user's history of likes can be viewed as a set of 'attributes' and has predictive quality for each user's personality traits (as measured by the BIG5 personality test). The 'likes' show affinity to certain topics and can therefore have predictive quality. As an example, a user that has 'liked' a Facebook page for 'meditation' or 'yoga' is probably likely to score high on 'openness' in the BIG5 personality test. The data for the 'likes' is very sparse, millions of pages have 
'likes' from users but on average for our dataset a user has 140 'likes'. Therefore only a limited subset of the 'likes' categories have any predictive value. Because of this inherent sparsity of the data, LASSO-regression [29] was used for prediction.

First a feature elimination is performed, where pages with less than 10 likes in total are removed, reducing the number of features from $10^{6}$ to $10^{4}$. This subset of features is then regressed on using lasso-regression. In order for the results to be comparable with those gathered in [7] the same methodology and metrics are used. 10-fold cross-validation is performed on the dataset, and the Pearson correlation coefficient is calculated for each split and then averaged over all the splits. For plotting, the data is partitioned based on how many 'likes' a user has, the correlation is plotted as a function of the number of 'likes' a user has. The average results over the 5 personality traits are depicted in Figure 3. The number of users that have 'likes' below 10 is small, so to estimate the predictive quality of users having 1 to 9 likes, we randomly query users with 10-20 'likes' and randomly subsample their likes to create user queries with the desired number of likes. The number of 'likes' a user has is a predictive indicator of personality, so to minimize this bias we restrict ourselves to query the users that are most similar in number of likes, i.e. the users that have 10-20 likes.

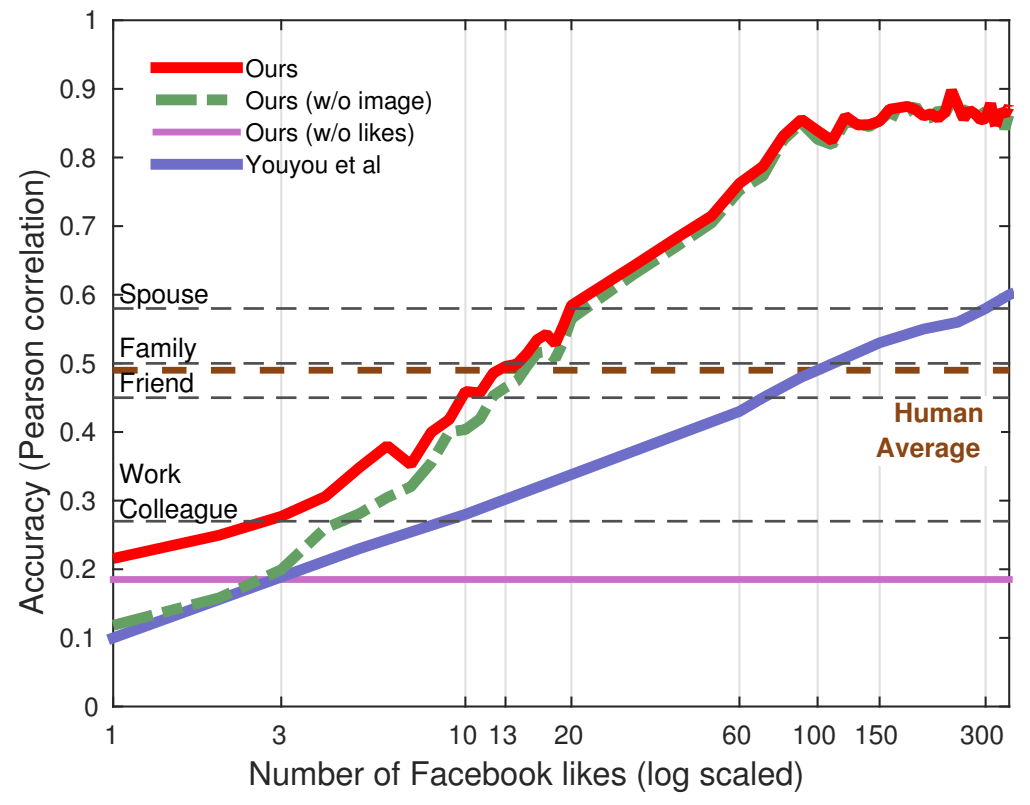

Fig. 3. Average accuracy of personality traits prediction. Human references included.

As shown in Figure 3 our average prediction of personality traits based solely on likes improves with the number of 'likes' available per user. This is expected as more information is known about the user. Note that our solution improves greatly over the results reported by Youyou et al. [7] for 4 or more likes, and 
that above 20 likes exceeds the performance of the spouse (0.58) to reach a plateau at more than 100 likes (0.86). Also, Youyou et al. LOWESS smooth and disattenuate their results, while we report the raw results. ${ }^{2}$

In the remainder of the paper, if not mentioned otherwise, we will use SVM prediction for CelebA experiments as the features are medium to low dimensional and the LASSO prediction is used for Facebook BIG5 due to the high dimensionality and sparsity of the data.

\begin{tabular}{|c|c|c|c|c|c|c|c|c|c|c|c|c|c|}
\hline Attribute & 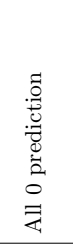 & 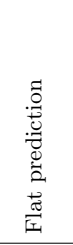 & 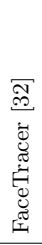 & 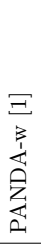 & 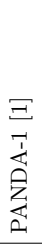 & 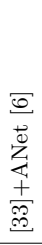 & 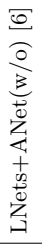 & 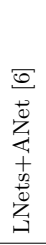 & 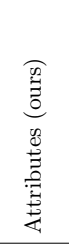 & 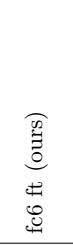 & 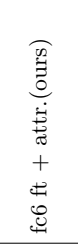 & 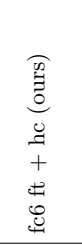 & 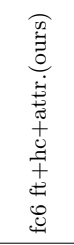 \\
\hline 5 o'Clock Shadow & 90.01 & 90.01 & 85 & 82 & 88 & 86 & 88 & 91 & 93.33 & 93.80 & 94.66 & 94.16 & 94.87 \\
\hline Arched Eyebrows & 71.56 & 71.56 & 76 & 73 & 78 & 75 & 74 & 79 & 80.59 & 79.91 & 83.39 & 80.99 & 84.08 \\
\hline Attractive & 50.42 & 50.42 & 78 & 77 & 81 & 79 & 77 & 81 & 78.20 & 81.04 & 81.87 & 82.10 & 82.62 \\
\hline Bags Under Eyes & 79.74 & 79.74 & 76 & 71 & 79 & 77 & 73 & 79 & 83.19 & 82.82 & 85.40 & 83.61 & 85.79 \\
\hline Bald & 97.88 & 97.88 & 89 & 92 & 96 & 92 & 95 & 98 & 97.89 & 98.80 & 98.85 & 98.72 & 98.78 \\
\hline Bangs & 84.43 & 84.43 & 88 & 89 & 92 & 94 & 92 & 95 & 84.45 & 94.42 & 94.55 & 95.02 & 95.14 \\
\hline Big Lips & 67.30 & 67.30 & 64 & 61 & 67 & 63 & 66 & 68 & 71.32 & 68.75 & 73.08 & 70.85 & 73.39 \\
\hline Big Nose & 78.80 & 78.80 & 74 & 70 & 75 & 74 & 75 & 78 & 83.55 & 81.95 & 84.57 & 82.12 & 85.35 \\
\hline Black Hair & 72.84 & 72.84 & 70 & 74 & 85 & 77 & 84 & 88 & 76.90 & 87.37 & 88.27 & 87.45 & 88.60 \\
\hline Blonde Hair & 86.67 & 86.67 & 80 & 81 & 93 & 86 & 91 & 95 & 86.65 & 95.30 & 95.63 & 95.33 & 95.77 \\
\hline Blurry & 94.94 & 94.94 & 81 & 77 & 86 & 83 & 80 & 84 & 94.94 & 95.91 & 96.04 & 96.13 & 96.24 \\
\hline Brown Hair & 82.03 & 82.03 & 60 & 69 & 77 & 74 & 78 & 80 & 82.44 & 86.61 & 87.44 & 87.22 & 88.04 \\
\hline Bushy Eyebrows & 87.05 & 87.05 & 80 & 76 & 86 & 80 & 85 & 90 & 87.28 & 89.32 & 89.55 & 90.65 & 90.75 \\
\hline Chubby & 94.70 & 94.70 & 86 & 82 & 86 & 86 & 86 & 91 & 95.78 & 95.03 & 95.95 & 95.26 & 96.16 \\
\hline Double Chin & 95.43 & 95.43 & 88 & 85 & 88 & 90 & 88 & 92 & 96.47 & 95.79 & 96.68 & 96.15 & 96.80 \\
\hline Eyeglasses & 93.54 & 93.54 & 98 & 94 & 98 & 96 & 96 & 99 & 93.56 & 98.69 & 98.72 & 98.92 & 98.89 \\
\hline Goatee & 95.42 & 95.42 & 93 & 86 & 93 & 92 & 92 & 95 & 95.83 & 96.77 & 97.08 & 96.93 & 97.15 \\
\hline Gray Hair & 96.81 & 96.81 & 90 & 88 & 94 & 93 & 93 & 97 & 96.90 & 97.95 & 98.03 & 98.16 & 98.29 \\
\hline Heavy Makeup & 59.50 & 59.50 & 85 & 84 & 90 & 87 & 85 & 90 & 88.75 & 89.92 & 91.19 & 90.61 & 91.78 \\
\hline High Cheekbones & 51.82 & 51.82 & 84 & 80 & 86 & 85 & 84 & 87 & 84.20 & 85.65 & 87.00 & 86.21 & 87.41 \\
\hline Male & 61.35 & 61.35 & 91 & 93 & 97 & 95 & 94 & 98 & 93.56 & 97.13 & 97.61 & 97.50 & 97.74 \\
\hline Mouth Slightly Open & 50.49 & 50.49 & 87 & 82 & 93 & 85 & 86 & 92 & 77.00 & 87.70 & 87.79 & 89.35 & 89.27 \\
\hline Mustache & 96.13 & 96.13 & 91 & 83 & 93 & 87 & 91 & 95 & 96.74 & 96.62 & 97.31 & 96.67 & 97.25 \\
\hline Narrow Eyes & 85.13 & 85.13 & 82 & 79 & 84 & 83 & 77 & 81 & 85.13 & 85.21 & 85.36 & 85.52 & 85.99 \\
\hline No Beard & 14.63 & 85.37 & 90 & 87 & 93 & 91 & 92 & 95 & 94.87 & 95.25 & 96.31 & 95.34 & 96.38 \\
\hline Oval Face & 70.44 & 70.44 & 64 & 62 & 65 & 65 & 63 & 66 & 75.83 & 74.06 & 77.71 & 75.00 & 78.33 \\
\hline Pale Skin & 95.79 & 95.79 & 83 & 84 & 91 & 89 & 87 & 91 & 95.79 & 95.81 & 95.83 & 96.71 & 96.81 \\
\hline Pointy Nose & 71.43 & 71.43 & 68 & 65 & 71 & 67 & 70 & 72 & 72.87 & 74.45 & 74.74 & 75.13 & 75.60 \\
\hline Receding Hairline & 91.51 & 91.51 & 76 & 82 & 85 & 84 & 85 & 89 & 91.65 & 92.13 & 92.52 & 92.54 & 92.67 \\
\hline Rosy Cheeks & 92.83 & 92.83 & 84 & 81 & 87 & 85 & 87 & 90 & 92.84 & 93.95 & 94.26 & 94.43 & 94.82 \\
\hline Sideburns & 95.36 & 95.36 & 94 & 90 & 93 & 94 & 91 & 96 & 96.04 & 97.30 & 97.53 & 97.54 & 97.58 \\
\hline Smiling & 49.97 & 50.03 & 89 & 89 & 92 & 92 & 88 & 92 & 84.76 & 91.59 & 92.22 & 92.05 & 92.65 \\
\hline Straight Hair & 79.01 & 79.01 & 63 & 67 & 69 & 70 & 69 & 73 & 79.29 & 82.59 & 83.00 & 82.58 & 83.21 \\
\hline Wavy Hair & 63.60 & 63.60 & 73 & 76 & 77 & 79 & 75 & 80 & 76.09 & 82.58 & 83.90 & 82.40 & 84.17 \\
\hline Wearing Earrings & 79.34 & 79.34 & 73 & 72 & 78 & 77 & 78 & 82 & 81.97 & 86.47 & 86.60 & 86.90 & 87.25 \\
\hline Wearing Hat & 95.80 & 95.80 & 89 & 91 & 96 & 93 & 96 & 99 & 95.79 & 98.45 & 98.59 & 98.76 & 98.89 \\
\hline Wearing Lipstick & 47.81 & 52.19 & 89 & 88 & 93 & 91 & 90 & 93 & 92.92 & 93.15 & 94.11 & 93.46 & 94.13 \\
\hline Wearing Necklace & 86.21 & 86.21 & 68 & 67 & 67 & 70 & 68 & 71 & 86.31 & 86.47 & 86.80 & 86.38 & 86.80 \\
\hline Wearing Necktie & 92.99 & 92.99 & 86 & 88 & 91 & 90 & 86 & 93 & 93.23 & 95.69 & 95.91 & 95.76 & 95.82 \\
\hline Young & 24.29 & 75.71 & 80 & 77 & 84 & 81 & 83 & 87 & 82.69 & 88.36 & 88.72 & 88.29 & 88.92 \\
\hline 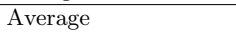 & 76.88 & 80.04 & 81 & 79 & 8 & 8 & 83 & 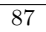 & 87.44 & 89.77 & 9062 & 90.22 & 91.00 \\
\hline
\end{tabular}

Table 2. CelebA prediction results (accuracy [\%]).

${ }^{2}$ Disattenuation always leads to equal or better results. 


\section{From face image to attribute prediction}

In this section we study attribute prediction task when starting from a face image, which is the traditional way of visual attribute prediction. In particular we evaluate different handcrafted and deep learned features on CelebA to then validate and report prediction results on both CelebA and Facebook BIG5 datasets. We used the off-the-shelf face detector of Mathias et al. [34] for detection and alignment of the faces as done for the DEX Network in $[11,12]$.

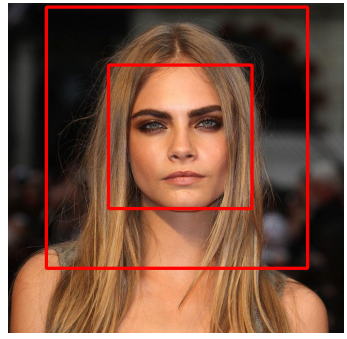

a) For the deep features descriptors the face is located and then a $40 \%$ padding is added around the face to get more context from the surrounding region

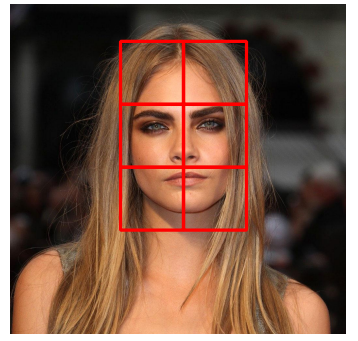

b) After the face is located c) Using relative location of facial it is split into blocks and handcrafted features are extracted for each block

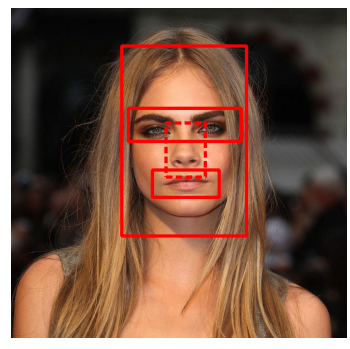

landmarks, regions of interest are extracted from the face and features extracted individually for each region

Fig. 4. Extraction sections for visual features.

\subsection{Handcrafted features.}

The recent years showed deep learned features to be generally more robust and to provide the best results for many computer vision tasks. However, they suffer from complexity as they usually require large datasets for training and have higher time and memory complexities for both training and testing than many handcrafted features (hc). Therefore, in the following we study three typical handcrafted features to embed the visual information from face images: LBP, SIFT and Color histograms. They are extracted in all the sections marked in Figure 4 and concatenated per each feature type.

Locally binary patterns (LBP) [35] were proposed by Ojala et al. For each pixel in the image, its surrounding pixels are thresholded based on the pixelvalue, which results in a binary number. A histogram of these numbers over an image or a section of an image is a powerful texture descriptor. In [35] a generalized gray-scale and rotation invariant version of the locally-binary-patterns is proposed, so called 'uniform' locally-binary-patterns. Here a 8 point LBP with radius 1 is used which results in a 10 bin histogram. This histogram is 12-normalized resulting in a 10-dimensional feature vector extracted for each section marked in Figure 4.

Scale-invariant feature transform (SIFT) For each section in Figure 4 a SIFT descriptor [36] is extracted. A neighborhood around the center of the image 
(or center of the image subsection) is taken. This neighborhood is divided into 16 sub-blocks and for each sub-block a 8 bin orientation histogram is created. This descriptor is then 12-normalized and used as a 128-dimensional feature vector.

Color histograms (Color) The color histograms have 64 uniform bins $(4 \times 4 \times 4)$ in the RGB color-space. These histograms are computed per each face image section and 12-normalized which results in a 64-dimensional feature vector.

\subsection{Deep features}

For the deep features, we extract the fc6 and fc7 4096-dimensional layers of the DEX Network [11], which is a variant of the VGG-16 [37] trained for age estimation on hundreds of thousands of face images from IMDB-WIKI dataset [11, 12]. Since most facial attributes are relevant signals for age estimation, these features work quite well off-the-shelf.

To further improve performance, we can finetune (ft) the network. However this poses a challenge, since attribute prediction is not a standard classification problem, but a multi-label one. While the network could be adapted for direct attribute prediction, it would result in a mismatch between the pre-trained network, trained for age classification, and the target task, attribute prediction. Instead, we cast the attribute prediction into a classification problem by predicting attribute configurations. To do this directly would be infeasible, since $L$ binary attributes have $2^{L}$ distinct possible configurations. However, due to the inter-dependencies between attributes, many of these configurations are unlikely to occur. We exploit this, and quantize the configuration space, by partitioning the training labels into $K$ clusters (using standard $k$-means clustering).

This transformation of the labels encodes the inter-dependencies of the attributes into a compact representation, for which we can learn fine-tuned features by treating as a a $K$-class classification task over the images. We thus only need to fine-tune the network once, obtaining a feature representation suitable for predicting jointly all the attributes, by discarding the last layer(s) of the network. For the final model, the quantized labels are discarded and an independent linear classifier is trained for each original attributes.

Compared to fine-tuning a separate binary classification network for each attribute, this reduces the fine-tuning time by a factor $L$. In all our fine-tuning experiments we set $K=50$, and iterate over 15000 minibatches with 10 images per batch, based on monitoring the validation error on a $90 \% / 10 \%$ split of the training data.

In the same way as for the handcrafted features, the deep features are 12normalized and when concatenated with other features, the total concatenation is also 12-normalized.

\subsection{Prediction results}

CelebA In Table 3 (w/o attributes) we report the performance on CelebA using various combinations of handcrafted and deep features extracted from the face 


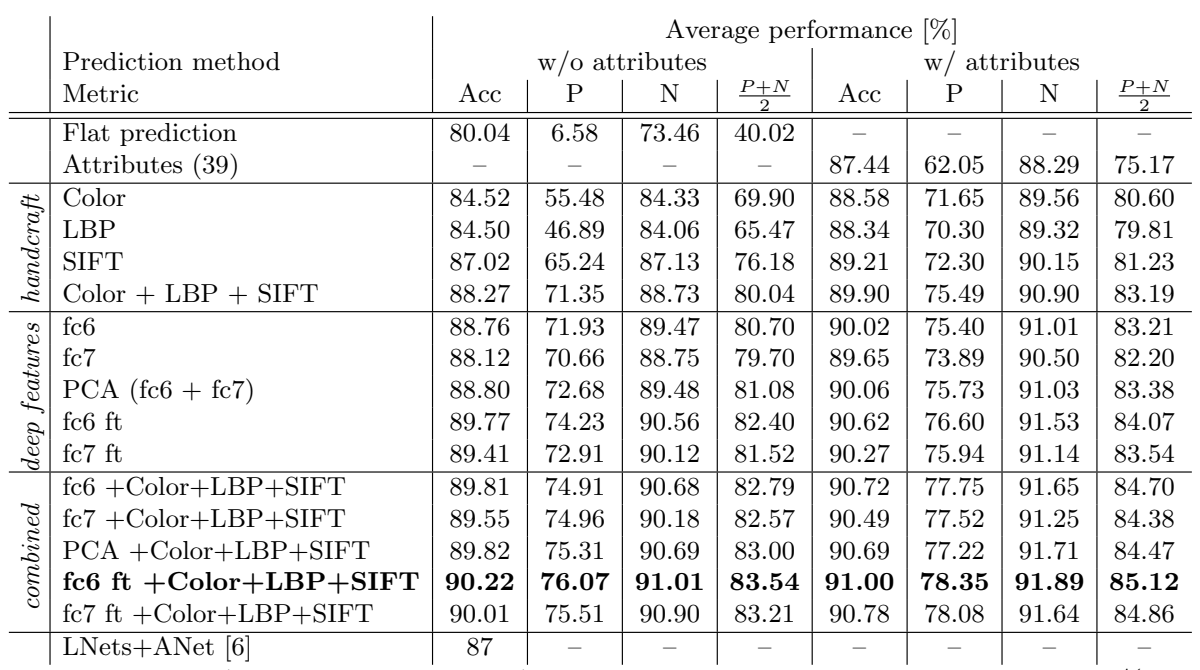

Table 3. CelebA prediction results. Acc: Standard accuracy. P: Positive rate $t p /(t p+$ $f p)$. N: Negative rate $t n /(t n+f n)$. Unbiased metric $(P+N) / 2$.

image. We report the numbers for each of these settings using standard accuracy, positive rate $P=t p /(t p+f p)$, negative rate $N=t n /(t n+f n)$ and the average $(P+N) / 2$, where $t p, f p$ are the number of true and false positives, and $t n, f n$ the number of true and false negatives. The average $(P+N) / 2$ provides an unbiased (ub.) metric for highly unbalanced class predictions as is the case for the CelebA dataset. The difference between the baseline and any of the classification methods becomes more pronounced and the numbers more illustrative of how the true predictions are split between the positive and the negative labels.

The focus in the discussion is on the accuracy numbers as they are the metric to compare to previous work but in brackets behind those numbers the unbiased metric will be reported.

Since most attributes in CelebA are unlikely to occur, the Flat prediction (that is predicting always the most often occurring 0 or 1 for each attribute) gives a powerful baseline of $80.04 \%(40.02 \%$ for ub. metric $(P+N) / 2)$. The handcrafted features (Color, LBP, SIFT) boost the performance significantly over Flat, with their combination reaching $88.27 \%$ ( $80.04 \%$ ub.). As often reported, using off-the-shelf deep features (Deep fc6 from DEX Network) beats the handcrafted ones by a small margin, $+0.49 \%(+0.66 \%$ ub.). Surprisingly though, the handcrafted and deep features are quite complementary, giving $89.81 \%(82.79 \%$ ub.) in combination.

The finetuned features (Deep fc6 - finetuned) significantly improve the performance, boosting the non-finetuned by $+1.01 \%$ (1.70\% ub.) and giving $90.22 \%$ $(83.54 \%$ ub.) in combination with the handcrafted ones. In our setups the deep fc6 features always led to better results than the deep fc7 features, while the combination of fc6 and fc7 features through a PCA projection preserving $99.9 \%$ of the energy just marginally improves over the results using fc6 alone. 
Facebook BIG5 In Figure 3 we show the accuracy of personality trait prediction on Facebook BIG5 dataset when using solely the profile image information, the 'Ours (w/o likes)' plot. It is remarkable that the performance of 0.18 , achieved using deep fc6 features from DEX Network, is better than that using solely up to 3 likes and not far from the work colleague reference of 0.27.

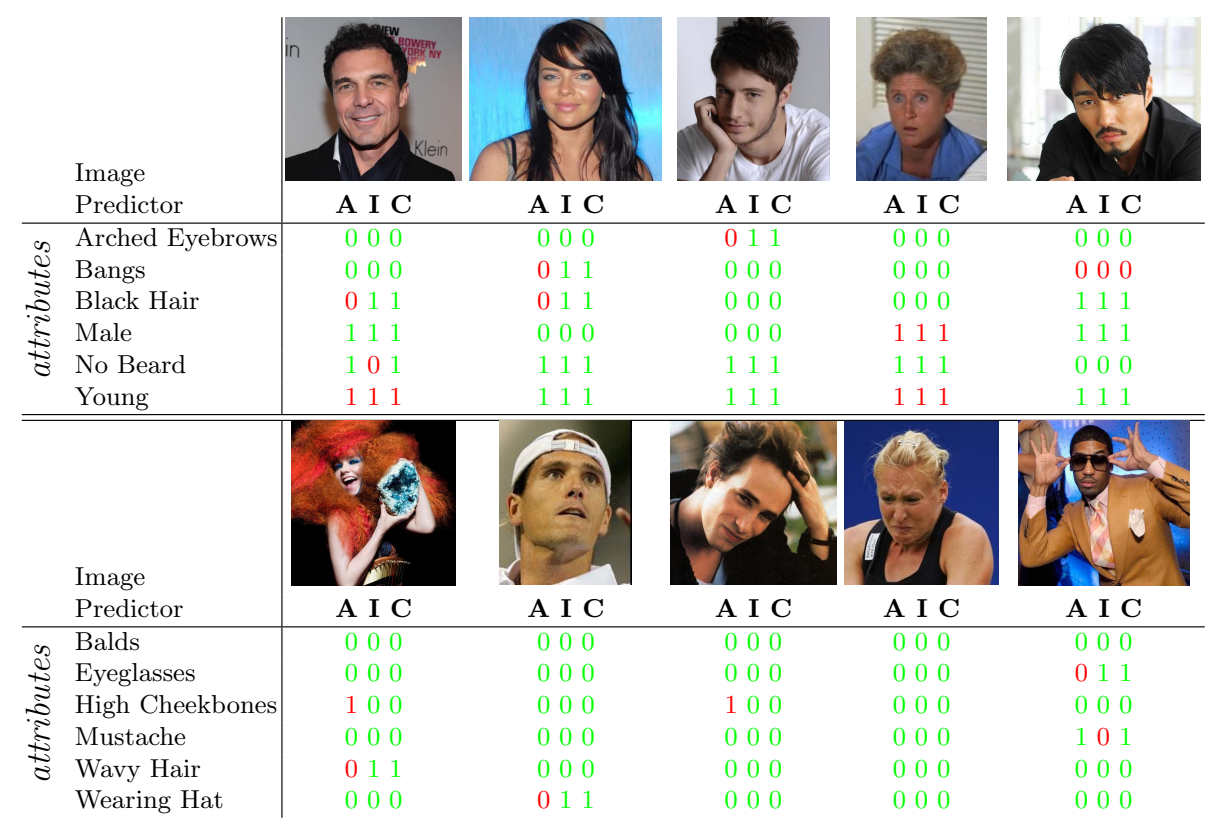

Fig. 5. Examples of correct (green) and wrong (red) attribute predictions from (A)ttributes, $(\mathbf{I})$ mages $(\mathrm{fc} 6 \mathrm{ft}+\mathrm{hc}$ ), and $(\mathbf{C})$ ombined attributes and images $(\mathrm{fc} 6 \mathrm{ft}$ $+\mathrm{hc}+$ attr).

\section{From face images and attributes to attributes}

In previous sections we achieved top attribute prediction accuracies when starting from either i) other attributes, or ii) handcrafted features and/or deep features extracted from face images. In this section we combine and discuss the combination of face features and attributes for improved attribute prediction.

CelebA The image features and the attributes are complimentary. Image features provide visual information while the attributes can provide information that is otherwise not easy to gather from images. Thus, we predict attributes by using image features and the rest of the attributes (concatenate image features with attributes). As can be seen in Table 2 (columns with +attr), concatenating the attributes with image features gives a boost to the average accuracy, up to $91.00 \%$ ( $85.12 \%$ ub.) when using the Deep fc6 and handcrafted features. In Figure 5 we show several examples of correct and wrong attribute prediction 
when starting from attributes (39), from image features ( $\mathrm{fc} 6 \mathrm{ft}+\mathrm{hc}$ ), and from the combined attributes and images $(\mathrm{fc} 6 \mathrm{ft}+\mathrm{hc}+$ attr). There are a couple of examples (Male, Bangs and Young attributes) where the failure is shared by all 3 predictors, while in most other cases at least 1 of the 3 predictors is correct.

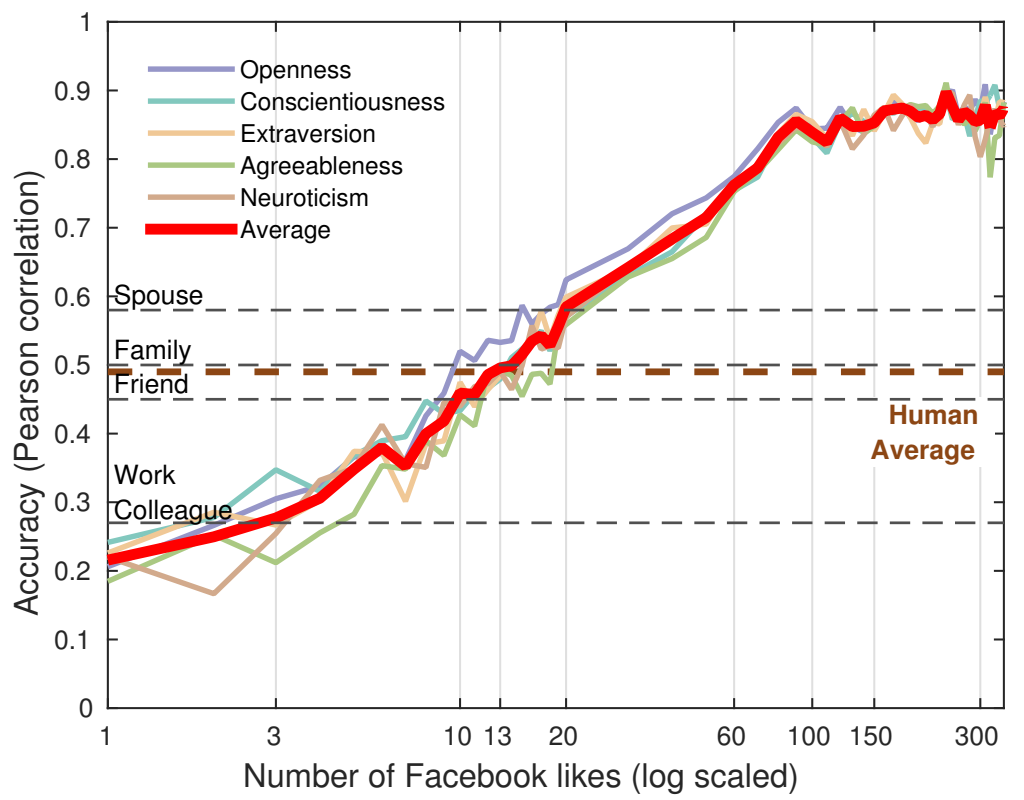

Fig. 6. Accuracy of personality traits prediction using 'likes' and image information.

Facebook BIG5 The likes provide a reliable way to predict the results of the BIG5 personality tests (in terms of Pearson correlation coefficient) but when the number of likes is reduced this prediction obviously breaks down. Therefore we employ the profile image as additional information to the likes (see Figure 3). In the 'region of few likes' the profile images from the Facebook BIG5 dataset provide a way to augment the prediction so that instead of starting from 0 , one can obtain a good prediction using only the image (a sound 0.18 Pearson correlation). When the number of likes increases the prediction becomes more reliable. With 3 likes and a profile face image the prediction performance surpasses the work colleague reference. With 10 likes and a picture we get comparable to the friend performance, while above 20 likes we exceed the spouse in predicting the personality traits of the Facebook user. Above 20 likes there is just a marginal benefit from using the profile image. Thus, the importance of the profile image is critical when very few or no likes are available and diminishes with the increase in the number of likes available for a user. 


\section{Conclusion}

In this paper we studied the prediction of attributes from other attributes, from face images, and from both attributes and face images. At the same time we analyzed handcrafted and deep visual features. Our validation was done on very recent large and complementary datasets: CelebA and Facebook BIG5. Our main findings are as follows:

1. The attributes to attribute prediction is a powerful technique not only on facial attribute prediction (CelebA) but also for predicting personality attributes based on sparse attributes such as likes in Facebook.

2. The handcrafted features, while a bit overlooked by the most recent works, are capable to capture the visual information and to lead to comparable performance to methods based on deep features. Furthermore, they are complementary when combined with the deep features.

3. The combination of visual and attribute information leads to better results than when using each individually.

4. For efficient deep learning we propose clustering of samples according to the attributes and, thus, achieve significant time savings when compared to per attribute training (a factor of 40 for CelebA).

5. We achieve state-of-the-art prediction for most facial attributes from CelebA.

6 . With as few as 20 likes we are able to predict as accurate as a spouse the personality traits for a Facebook user, with only 3 likes and a profile picture we reach the level of accuracy of a work colleague, while at 100 likes we achieve a 0.86 Pearson correlation of the prediction with the ground truth.

7. We require 3 up to 10 times fewer likes for better BIG5 prediction accuracy when compared with previous results reported on Facebook BIG5 dataset.

Some observations are harsh but necessary:

i) The flat prediction, without even touching the test samples, is comparable to a number of (involved) methods from the literature.

ii) Common standard handcrafted features combined with standard linear classifiers can achieve top performance comparable or better than existing deep learned top methods, and are complementary to the deep features.

iii) People are amazingly easy to 'read' by knowing just a profile picture and/or a couple of likes in a social network as shown by our experiments.

As future work we plan to further explore the relationships between images, attributes and any other information connected to an individual, object, or simple conceptualized instance and to be able to bridge them such that to not only predict attributes but to embed knowledge and transfer it.

Acknowledgement. This work was supported by the ETH General Fund (OK) and by a K40 GPU grant from NVidia. We thank Michal Kosinski and David Stillwell for providing the Facebook BIG5 dataset. 


\section{References}

1. Zhang, N., Paluri, M., Ranzato, M., Darrell, T., Bourdev, L.: Panda: Pose aligned networks for deep attribute modeling. In: The IEEE Conference on Computer Vision and Pattern Recognition (CVPR). (2014)

2. Liu, J., Kuipers, B., Savarese, S.: Recognizing human actions by attributes. In: Computer Vision and Pattern Recognition (CVPR), 2011 IEEE Conference on. (2011) 3337-3344

3. Kumar, N., Berg, A., Belhumeur, P.N., Nayar, S.: Describable visual attributes for face verification and image search. IEEE Transactions on Pattern Analysis and Machine Intelligence 33 (2011) 1962-1977

4. Layne, R., Hospedales, T.M., Gong, S.: Person re-identification by attributes. In: BMVC. (2012)

5. Siddiquie, B., Feris, R.S., Davis, L.S.: Image ranking and retrieval based on multiattribute queries. In: Computer Vision and Pattern Recognition (CVPR), 2011 IEEE Conference on. (2011) 801-808

6. Liu, Z., Luo, P., Wang, X., Tang, X.: Deep learning face attributes in the wild. In: The IEEE International Conference on Computer Vision (ICCV). (2015)

7. Youyou, W., Kosinski, M., Stillwell, D.: Computer-based personality judgments are more accurate than those made by humans. Proceedings of the National Academy of Sciences 112 (2015) 1036-1040

8. Ciresan, D., Meier, U., Schmidhuber, J.: Multi-column deep neural networks for image classification. In: Computer Vision and Pattern Recognition (CVPR), 2012 IEEE Conference on. (2012) 3642-3649

9. Krizhevsky, A., Sutskever, I., Hinton, G.E.: Imagenet classification with deep convolutional neural networks. In Pereira, F., Burges, C.J.C., Bottou, L., Weinberger, K.Q., eds.: Advances in Neural Information Processing Systems 25. Curran Associates, Inc. (2012) 1097-1105

10. He, K., Zhang, X., Ren, S., Sun, J.: Deep residual learning for image recognition. In: Computer Vision and Pattern Recognition (CVPR), 2016 IEEE Conference on. (2016)

11. Rothe, R., Timofte, R., Van Gool, L.: Dex: Deep expectation of apparent age from a single image. In: The IEEE International Conference on Computer Vision (ICCV) Workshops. (2015)

12. Rothe, R., Timofte, R., Van Gool, L.: Deep expectation of real and apparent age from a single image without facial landmarks. International Journal and Computer Vision (IJCV) (2016)

13. Rothe, R., Timofte, R., Van Gool, L.: Some like it hot - visual guidance for preference prediction. In: Computer Vision and Pattern Recognition (CVPR), 2016 IEEE Conference on. (2016)

14. Uricar, M., Timofte, R., Rothe, R., Matas, J., Van Gool, L.: Structured Output SVM Prediction of Apparent Age, Gender and Smile From Deep Features. In: Computer Vision and Pattern Recognition (CVPR), Workshops. (2016)

15. Kosinski, M., Stillwell, D., Graepel, T.: Private traits and attributes are predictable from digital records of human behavior. Proceedings of the National Academy of Sciences 110 (2013) 5802-5805

16. Tsoumakas, G., Katakis, I.: Multi-label classification: An overview. Dept. of Informatics, Aristotle University of Thessaloniki, Greece (2006)

17. Fürnkranz, J., Hüllermeier, E., LozaMencía, E., Brinker, K.: Multilabel classification via calibrated label ranking. Machine Learning 73 (2008) 133-153 
18. Boutell, M.R., Luo, J., Shen, X., Brown, C.M.: Learning multi-label scene classification. Pattern Recognition 37 (2004) 1757 - 1771

19. Read, J., Pfahringer, B., Holmes, G., Frank, E.: Classifier chains for multi-label classification. Machine Learning 85 (2011) 333-359

20. Akata, Z., Reed, S., Walter, D., Lee, H., Schiele, B.: Evaluation of output embeddings for fine-grained image classification. In: The IEEE Conference on Computer Vision and Pattern Recognition (CVPR). (2015)

21. Caruana, R.: Multitask learning. Machine Learning 28 (1997) 41-75

22. Wang, G., Forsyth, D.: Joint learning of visual attributes, object classes and visual saliency. In: 2009 IEEE 12th International Conference on Computer Vision. (2009) $537-544$

23. Wang, Y., Mori, G.: A Discriminative Latent Model of Object Classes and Attributes. In: Computer Vision - ECCV 2010: 11th European Conference on Computer Vision, Heraklion, Crete, Greece, September 5-11, 2010, Proceedings, Part V. Springer Berlin Heidelberg, Berlin, Heidelberg (2010) 155-168

24. Parikh, D., Grauman, K.: Relative attributes. In: 2011 International Conference on Computer Vision. (2011) 503-510

25. Lampert, C.H., Nickisch, H., Harmeling, S.: Learning to detect unseen object classes by between-class attribute transfer. In: Computer Vision and Pattern Recognition, 2009. CVPR 2009. IEEE Conference on. (2009) 951-958

26. Palatucci, M., Pomerleau, D., Hinton, G.E., Mitchell, T.M.: Zero-shot learning with semantic output codes. In Bengio, Y., Schuurmans, D., Lafferty, J.D., Williams, C.K.I., Culotta, A., eds.: Advances in Neural Information Processing Systems 22. Curran Associates, Inc. (2009) 1410-1418

27. Costa, P.T., McCrae, R.R.: Revised NEO personality inventory (NEO PI-R) and NEP five-factor inventory (NEO-FFI): professional manual. Psychological Assessment Resources Lutz, FL (1992)

28. Goldberg, L.R., Johnson, J.A., Eber, H.W., Hogan, R., Ashton, M.C., Cloninger, C.R., Gough, H.G.: The international personality item pool and the future of public-domain personality measures. Journal of Research in Personality 40 (2006) 84 - 96 Proceedings of the 2005 Meeting of the Association of Research in PersonalityAssociation of Research in Personality.

29. Tibshirani, R.: Regression shrinkage and selection via the lasso. Journal of the Royal Statistical Society. Series B (Methodological) 58 (1996) 267-288

30. Cortes, C., Vapnik, V.: Support-vector networks. Machine Learning 20 (1995) 273-297

31. Chang, C.C., Lin, C.J.: Libsvm: A library for support vector machines. ACM Trans. Intell. Syst. Technol. 2 (2011) 27:1-27:27

32. Kumar, N., Belhumeur, P., Nayar, S.: FaceTracer: A Search Engine for Large Collections of Images with Faces. In: Computer Vision - ECCV 2008: 10th European Conference on Computer Vision, Marseille, France, October 12-18, 2008, Proceedings, Part IV. Springer Berlin Heidelberg, Berlin, Heidelberg (2008) 340-353

33. Li, J., Zhang, Y.: Learning surf cascade for fast and accurate object detection. In: Computer Vision and Pattern Recognition (CVPR), 2013 IEEE Conference on. (2013) 3468-3475

34. Mathias, M., Benenson, R., Pedersoli, M., Van Gool, L.: Face Detection without Bells and Whistles. In: Computer Vision - ECCV 2014: 13th European Conference, Zurich, Switzerland, September 6-12, 2014, Proceedings, Part IV. Springer International Publishing, Cham (2014) 720-735 
35. Ojala, T., Pietikainen, M., Maenpaa, T.: Multiresolution gray-scale and rotation invariant texture classification with local binary patterns. IEEE Transactions on Pattern Analysis and Machine Intelligence 24 (2002) 971-987

36. Lowe, D.G.: Object recognition from local scale-invariant features. In: Proceedings of the International Conference on Computer Vision-Volume 2 - Volume 2. ICCV '99, Washington, DC, USA, IEEE Computer Society (1999) 1150-

37. Simonyan, K., Zisserman, A.: Very deep convolutional networks for large-scale image recognition. CoRR abs/1409.1556 (2014) 